\title{
Bradycardia in a patient with lung cancer
}

A 70-year-old man presented with fatigue and dyspnea. He denied any chest pain, orthopnea, pedal oedema, dizziness and syncope. He had a history of hypertension, hyperlipidaemia and minor coronary artery disease identified on coronary angiography performed a year ago. He also had metastatic squamous cell lung carcinoma and last received carboplatin, paclitaxel and a second cycle of pembrolizumab a month before presenting. His blood pressure was $115 / 55 \mathrm{mmHg}$, pulse rate was 38 beats/min and oxygen saturation was $96 \%$ on room air. He was afebrile. Laboratory results revealed markedly raised troponin I of $18,266 \mathrm{ng} / \mathrm{L}$ (normal $<17.4 \mathrm{ng} / \mathrm{L}$ ), N-terminal pro-brain natriuretic peptide (NT-proBNP) of $8140 \mathrm{pg} /$ $\mathrm{mL}$ (normal $<241 \mathrm{pg} / \mathrm{mL}$ ) and creatine kinase of 4789U/L (normal 30-350U/L). An electrocardiogram (ECG) was obtained (Fig. 1).

The ECG demonstrates complete heart block with a ventricular escape rate of 31 beats $/ \mathrm{min}$. There are irregular PR intervals with constant P-P and R-R intervals consistent with atrioventricular dissociation. The broad QRS complexes suggest an infra-Hisian escape rhythm with the level of block below the bundle of His. The patient's baseline ECG showed normal sinus rhythm with narrow QRS complexes.

What is the most likely cause of this patient's complete heart block given his medical history and clinical presentation?
A. Acute myocardial infarction
B. Beta blocker therapy
C. Myocarditis
D. Lyme disease
E. Degeneration of conduction system

Clinical course. The absence of symptoms of myocardial ischaemia and/or ST segment changes on the ECG makes option A unlikely. A careful review of the patient's medication list did not reveal atrioventricular nodal blocking agents, ruling out option $\mathrm{B}$ as an aetiology. Travel history was negative for Lyme disease-endemic areas, and in the absence of typical systemic symptoms and physical signs, option D is highly unlikely. Option E is possible given the patient's age; however, given his past medical history, other reversible aetiologies need to be evaluated for in this clinical context.

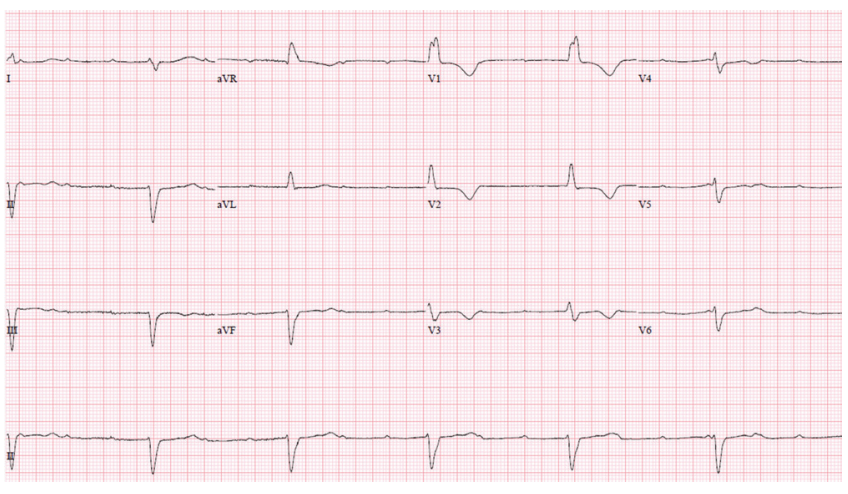

Fig. 1. Twelve-lead electrocardiogram (ECG) at presentation.

The patient was transferred to the coronary care unit for insertion of a temporary pacing wire. Transthoracic echocardiogram (TTE) revealed global left ventricular hypokinesia with a left ventricular ejection fraction of $42 \%$. This was a drop from $60 \%$ demonstrated on a TTE performed prior to the start of chemotherapy treatment. Serum aldolase was raised at $71.3 \mathrm{U} / \mathrm{L}$ (normal $<7.6 \mathrm{U} / \mathrm{L}$ ). The cardio-oncology team was consulted and the diagnosis was refined to immunerelated adverse events (irAEs) from pembrolizumab, an immune checkpoint inhibitor (ICI), resulting in complete heart block secondary to myocarditis (option C). There was also concurrent ICI-mediated myositis. The patient underwent an endomyocardial biopsy and was treated with empirical high-dose intravenous methylprednisolone 1 gram/day for 5 days before conversion to oral prednisolone. Serum cardiac biomarkers and muscle enzyme levels significantly reduced with steroid therapy. The patient experienced an episode of mild fluid overload that improved with oral diuretics. However, he required a permanent pacemaker implantation as he remained pacing dependent despite a week of steroid treatment. Endomyocardial biopsy showed inflammatory cell infiltration and myocardial necrosis with increased programmed cell death-ligand 1 (PD-L1) immunoexpression, confirming the diagnosis of ICI-mediated myocarditis (Fig. 2). The patient improved after pacemaker implantation and was discharged stable.

Discussion. ICI-mediated myocarditis is a rare but notable cause of complete heart block which may be misdiagnosed. Common causes of complete heart block include degeneration of the conduction system, acute myocardial infarction and atrioventricular nodal 


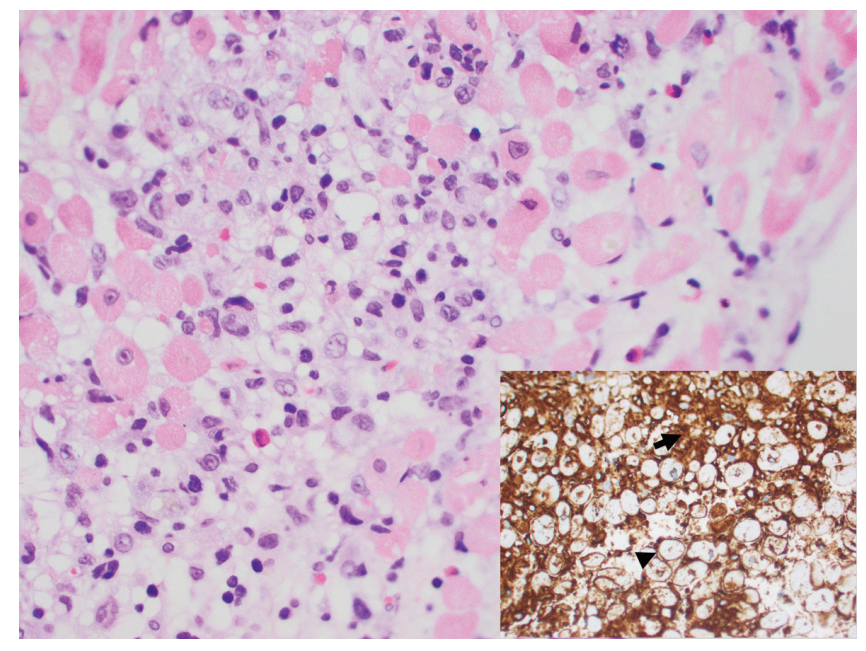

Fig. 2. Photomicrograph of endomyocardial biopsy showing area of myocardial necrosis with inflammatory infiltrate comprising histiocytes, lymphocytes and eosinophils. Inset: Positive immunoexpression of PD-L1 in inflammatory cells (arrow) and cardiomyocytes (arrowhead). (Main image: haematoxylin and eosin stain. Inset: immunoperoxidase stain. Both images: magnification $\mathrm{x} 400$.)

PD-L1: programmed cell death-ligand 1

blocking agents. Management involves addressing the underlying cause and supportive treatment via advanced cardiac life support algorithms.

ICIs are an emerging class of cancer therapy agents used in the treatment of solid and haematological malignancies. One example of an ICI is pembrolizumab, which is a programmed cell death protein 1 (PD-1) inhibitor. Multiple unique irAEs from ICI therapy have been described. They typically occur after $2-3$ cycles of ICI therapy, and most commonly involve the skin and gastrointestinal system. ${ }^{1}$ Although cardiac irAEs are rare with an incidence of approximately $1 \%$, they are associated with high mortality of $25-50 \% .{ }^{2}$ Cardiac irAEs include myocarditis, atrioventricular block, ventricular dysrhythmias, heart failure and pericarditis. ${ }^{2}$ The diagnosis of ICI-mediated myocarditis is supported by elevated troponins, conduction abnormalities or ischaemic changes on ECG, left ventricular dysfunction or wall motion abnormalities on TTE, and myocardial oedema or late gadolinium enhancement on cardiac magnetic resonance imaging (MRI). ${ }^{3}$ Endomyocardial biopsy is the gold standard for diagnosis, and findings include inflammatory cell infiltration and myocardial necrosis. Up-regulation and positive immunostaining of PD-L1 have been described in ICI-mediated myocarditis. ${ }^{3}$ Clinicians should be aware of the triad of myocarditis, myositis and myasthenia gravis as it is associated with poorer outcomes. ${ }^{4}$ Risk factors for irAEs include dual ICI therapy and pre-existing cardiovascular or autoimmune disease. ${ }^{2}$
The American Society of Clinical Oncology (ASCO) Clinical Practice Guideline ${ }^{5}$ recommends baseline ECG and troponins especially for patients treated with combination immunotherapies, and further testing upon the development of signs and symptoms. Additional testing may include TTE, chest X-ray, stress testing and cardiac MRI as guided by cardio-oncology. ASCO also recommends permanent discontinuation of ICI in the event of grade 1 cardiovascular toxicity (defined by abnormal cardiac biomarker levels and/or ECG). The cardio-oncology unit at the Vanderbilt University Medical Center, US recommends weekly surveillance of troponin levels for 6 weeks after initiation of ICI. ${ }^{6}$ Patients with raised troponins prior to the initiation of ICI are assumed to have injury unrelated to ICI treatment, while those with troponin elevation on surveillance should be referred to cardio-oncology for evaluation of myocarditis and concomitant myositis.

Patients with irAEs often present with non-specific symptoms and may mimic common conditions such as acute myocardial infarction or sepsis. Emergency medicine and internal medicine physicians are usually on the front line of these acute presentations. Hence, a high level of suspicion and early recognition is crucial to institute prompt and appropriate treatment. ${ }^{1}$ Management of cardiac irAEs includes discontinuation of ICI therapy and early consultation with the cardio-oncology team. Treatment of ICI-mediated myocarditis involves early high-dose intravenous methylprednisolone until the patient is clinically stable, followed by oral prednisolone tapered over weeks. ${ }^{7}$ Second-line agents include mycophenolate mofetil or infliximab. Atrioventricular block may improve with discontinuation of ICI and institution of steroid treatment, although permanent pacemaker insertions have been required. ${ }^{3}$

\section{REFERENCES}

1. Shah M, Rajha E, DiNardo C, et al. Adverse Events of Novel Therapies for Hematologic Malignancies: What Emergency Physicians Should Know. Ann Emerg Med 2020;75:264-86.

2. Lyon AR, Yousaf N, Battisti NML, et al. Immune checkpoint inhibitors and cardiovascular toxicity. Lancet Oncol 2018; 19:e447-58

3. Palaskas N, Lopez-Mattei J, Durand JB, et al. Immune Checkpoint Inhibitor Myocarditis: Pathophysiological Characteristics, Diagnosis, and Treatment. J Am Heart Assoc 2020;9:e013757.

4. Kimura T, Fukushima S, Miyashita A, et al. Myasthenic crisis and polymyositis induced by one dose of nivolumab. Cancer Sci 2016;107:1055-8.

5. Brahmer JR, Lacchetti C, Schneider BJ, et al. Management of immune-related adverse events in patients treated with immune checkpoint inhibitor therapy: American Society of Clinical Oncology Clinical Practice Guideline. J Clin Oncol 2018;36:1714-68. 
6. Hu JR, Florido R, Lipson EJ, et al. Cardiovascular toxicities associated with immune checkpoint inhibitors. Cardiovascular Res 2019;115:854-68.

7. Zhang L, Zlotoff DA, Awadalla M, et al. Major Adverse Cardiovascular Events and the Timing and Dose of Corticosteroids in Immune Checkpoint Inhibitor-Associated Myocarditis. Circulation 2020;141:2031-4.

Zhe Yan $\underline{\mathrm{Ng}}{ }^{1}{ }_{\text {MRCP (UK) }}$, Chieh Yang $\underline{\operatorname{KoO}}{ }^{1,2}{ }_{\text {MRCP (UK) }}$, Kong Bing Tan ${ }^{2,3}$ FRCPath, Weiqin Lin ${ }^{1,2}{ }_{\text {MRCP (UK), }}$ Matilda Lee ${ }^{4}{ }_{M R C P}(U K)$, Li Ling Tan ${ }^{1,2}{ }_{M R C P}(U K)$
${ }^{1}$ Department of Cardiology, National University Heart Centre Singapore, Singapore

${ }^{2}$ Yong Loo Lin School of Medicine, National University of Singapore, Singapore

${ }^{3}$ Department of Pathology, National University Hospital, Singapore

${ }^{4}$ Department of Haematology-Oncology, National University Cancer Institute, Singapore

Correspondence: Dr Zhe Yan Ng, Department of Cardiology, National University Heart Centre, Singapore, 1E Kent Ridge Road, NUHS Tower Block, Level 9, Singapore 119228.

Email: zhe_yan_ng@nuhs.edu.sg 